\title{
Verteporfin enhances the sensitivity of LOVO/TAX cells to taxol via YAP inhibition
}

\author{
GANGGANG SHI $^{1}$, HAO WANG $^{2}$, HONGQIU HAN $^{2}$, JIANCHEN GAN ${ }^{1}$ and HUI WANG ${ }^{1}$ \\ ${ }^{1}$ Department of Colorectal Surgery, The Second Hospital of Tianjin Medical University, Tianjin 300211; \\ ${ }^{2}$ Department of General Surgery, Tianjin Medical University General Hospital, Tianjin 300052, P.R. China
}

Received January 6, 2017; Accepted August 22, 2018

DOI: $10.3892 /$ etm.2018.6447

\begin{abstract}
Studies have reported that taxol (TAX) is an effective drug for the treatment of colorectal cancer; however, its application inevitably results in drug resistance. Overexpression of Yes-associated protein (YAP) is considered one of the factors that cause TAX resistance, which may be inhibited by verteporfin (VP) treatment. The present study aimed to confirm the role of YAP in TAX resistance and to investigate whether the drug sensitivity of the TAX-resistant LOVO/TAX cell line to TAX is affected by VP treatment. The role of YAP in TAX resistance was first determined through vector-mediated overexpression and inhibition of YAP in cells. Reverse-transcription quantitative polymerase chain reaction and western blot analysis were performed for detection of associated mRNA and protein, respectively. An MTT assay was used to detect the drug sensitivity of cells to TAX. The results suggested that compared with that in the native LOVO cell line, YAP expression was significantly increased in LOVO/TAX cells. YAP gene silencing markedly enhanced the drug sensitivity of LOVO/TAX cells to TAX and, on the contrary, the drug sensitivity notably declined when YAP was overexpressed in LOVO cells. The results indicated that YAP gene expression and TAX resistance were correlated. VP treatment suppressed YAP expression and increased the drug sensitivity of LOVO/TAX cells to TAX in a dose-dependent manner. In addition, compared with VP alone, VP and TAX combination therapy had a greater inhibitory effect on YAP expression. VP treatment enhanced the drug sensitivity of LOVO/TAX cells to TAX through inhibiting YAP expression.
\end{abstract}

Correspondence to: Dr Hui Wang, Department of Colorectal Surgery, The Second Hospital of Tianjin Medical University, 23 Pingjiang Street, Hexi, Tianjin 300211, P.R. China

E-mail:wanghui170106@sina.com

Key words: colorectal cancer, taxol, verteporfin, drug-sensitivity, Yes-associated protein

\section{Introduction}

Colorectal carcinoma (CRC) is the third most frequent cancer in males and the second most frequent in females worldwide. Approximately 1.4 million cases were newly diagnosed and $\sim 700,000$ patients died from CRC in 2012 (1). Tumor progression and metastasis were the major causes of the death of CRC patients. At present, surgery and chemotherapy or Chinese medicine treatment are the major clinical treatments for CRC (2). However, although surgery is an effective method for CRC treatment, total resection of the lesion is not possible and chemotherapeutic treatment for CRC frequently results in drug resistance, which currently represents a challenge in CRC treatment.

Yes-associated protein (YAP) is a key effective factor of the Hippo signaling pathway. As a newly discovered cell signal transduction pathway, the Hippo signaling pathway has important roles in regulating the volume and size of organs $(3,4)$. YAP upregulation has been identified in various cancer types, including ovarian, lung, liver, gastric and prostate cancer and it has been associated with tumor progression and sensitivity to chemotherapy, indicating that YAP participates in tumor development (5-9). YAP has been reported to contribute to the resistance of various cancer types to chemotherapy drugs (10).

As one of the most effective anti-tumor agents, taxol (TAX) has been deeply researched and widely used for cancer treatment, particularly of ovarian and breast cancers. However, its function is often limited by drug resistance. Studies have indicated that the function of YAP as a transcription factor may be suppressed by TAX and YAP upregulation reverses this suppression (11-13).

Verteporfin (VP), a member of the porphyrin family, is clinically used in the treatment of neovascular macular degeneration (14). It has been suggested that VP may suppress hepatoma cell growth without photo-activation through suppressing the YAP-TEA domain protein (TEAD) complex (15). Thus, the present study hypothesized that YAP suppression caused by VP treatment enhances the drug sensitivity of TAX-resistant colorectal cancer cells to TAX, which may provide a novel scientific approach for improving the clinical application of TAX.

The present study aimed to investigate whether the sensitivity of the TAX-resistant colorectal cancer cell line LOVO (LOVO/TAX) to TAX may be enhanced by VP treatment. 


\section{Materials and methods}

Cells and cell culture. The LOVO cell line and the LOVO/TAX cell line were purchased from Shanghai Bogoo Biotechnology Co., Ltd. (Shanghai, China). LOVO and LOVO/TAX cells were cultured in Dulbecco's modified Eagle's medium (DMEM; Gibco; Thermo Fisher Scientific, Inc., Waltham, MA, USA) supplemented with $10 \%$ fetal bovine serum (FBS; Gibco; Thermo Fisher Scientific, Inc.), 1\% penicillin and streptomycin solution and were incubated at $37^{\circ} \mathrm{C}$ with $5 \% \mathrm{CO}_{2}$. Cells were passaged until reaching at least $90 \%$ confluence.

Determination of drug sensitivity by MTT assay. Cells were seeded into a 96-well plate and cultured in DMEM media for $24 \mathrm{~h}$. The cells were then treated with various concentrations of TAX for $48 \mathrm{~h}$. Subsequently, $10 \mu \mathrm{l} \mathrm{MTT}$ solution $(5 \mathrm{mg} / \mathrm{ml})$ was added to each well, followed by incubation at $37^{\circ} \mathrm{C}$ for $4 \mathrm{~h}$. The formazan crystals were subsequently dissolved with dimethylsulfoxide. The optical absorbance was measured at $450 \mathrm{~nm}$ using a spectrophotometer (Bio-Tek Instruments, Winooski, VT, USA). Each experiment was repeated three times.

Cell transfection. To explore the association between YAP and TAX resistance, YAP expression plasmid, YAP small interfering (si)RNA, YAP expression control and YAP siRNA control plasmid (Vigene Bioscience, Inc., Rockville, MD, USA) were respectively transfected into LOVO cells or LOVO/TAX cells with $30 \mu$ l Lipofectamine 2000 transfection reagent (Invitrogen; Thermo Fisher Scientific, Inc.) according to the manufacturer's instructions. After $24 \mathrm{~h}$ of incubation, the transfected cells were subjected to the following analyses.

Western blot analysis. For protein detection, western blot analysis was performed. In short, radioimmunoprecipitation assay buffer (Solar Bio, Beijing, China) supplemented with $1 \mathrm{mM}$ phenylmethanesulfonyl fluoride was used for cell protein extraction. Subsequently, the protein concentration was determined using a bicinchoninic acid assay. Proteins ( $2 \mu \mathrm{g} / \mathrm{lane})$ were separated by $10 \%$ SDS-PAGE and then transferred onto polyvinylidene difluoride membranes (EMD Millipore, Billerica, MA, USA). Membranes were blocked at room temperature for $1 \mathrm{~h}$ in PBS containing $0.1 \%$ Tween- 20 and $5 \%$ fat-free powdered milk. Membranes were then incubated overnight at $4^{\circ} \mathrm{C}$ with primary antibodies [anti- $\beta$-actin (cat. no. 4970; 1:1,000); anti-YAP (cat. no. 14074; 1:1,000); anti-B-cell lymphoma 2 (Bcl-2; cat. no. 3498; 1:1,000); anti-Bcl-2-associated X protein (Bax; cat. no. 5023; 1:1,000; all Cell Signaling Technology Inc., Danvers, MA, USA)] followed by incubation with a secondary antibody (cat. no. 7074; 1:5,000; Cell Signaling Technology Inc.) at room temperature for $2 \mathrm{~h}$. An enhanced chemiluminescence kit (PE0010; Beijing Solarbio Science \& Technology Co., Ltd., Beijing, China) was used to detect the protein bands and images were captured by ImageJ (v1.4.1; National Institutes of Health, Bethesda, MD, USA).

RNA isolation and reverse-transcription quantitative polymerase chain reaction ( $R T-q P C R)$. Total RNA from cells was extracted with TRIzol reagent (Invitrogen; Thermo Fisher Scientific, Inc.) according to the manufacturer's instructions. Complementary DNA was generated by using the PrimeScript $^{\mathrm{TM}}$ RT reagent kit (Takara Bio, Inc., Otsu, Japan).
Table I. Primer sequences for polymerase chain reaction.

\begin{tabular}{lc}
\hline Gene/direction & Sequence (5'-3') \\
\hline YAP & \\
Forward & ACGTTCATCTGGGACAGCAT \\
Reverse & GTTGGGAGATGGCAAAGACA \\
GAPDH & \\
Forward & CTTTGGTATCGTGGAAGGACTC \\
Reverse & GTAGAGGCAGGGATGATGTTCT
\end{tabular}

YAP, Yes-associated protein.

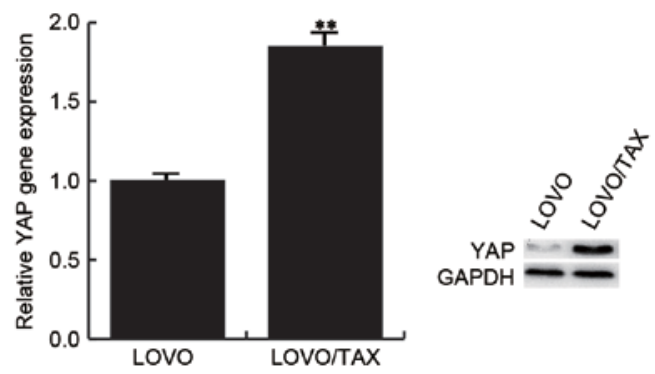

Figure 1. YAP expression in native and taxol-resistant cells. The expression of YAP in LOVO and LOVO/TAX cells was detected by reverse-transcription quantitative polymerase chain reaction and western blot analysis, respectively. Values are expressed as the mean \pm standard deviation. ${ }^{* *} \mathrm{P}<0.01$. YAP, Yes-associated protein; LOVO/TAX, taxol-resistant LOVO cell line.

Real-time PCR was performed by using the SYBR ${ }^{\circledR}$ Premix Ex $\mathrm{Taq}^{\mathrm{TM}}$ II (Takara Bio, Inc.) and all reactions were performed in triplicate under the following conditions: $95^{\circ} \mathrm{C}$ for $10 \mathrm{~min}$, followed by 37 cycles at $95^{\circ} \mathrm{C}$ for $15 \mathrm{sec}$ and $72^{\circ} \mathrm{C}$ for $30 \mathrm{sec}$. GAPDH was used as an internal control. The $2^{-\Delta \Delta C a}$ method was used for calculation of the relative gene expression levels (16). The primers used for real-time PCR are listed in Table I.

Statistical analysis. Experiments were performed for at least three times. SPSS 17.0 statistical software (SPSS, Inc., Chicago, IL, USA) was used for all statistical analyses. Values are expressed as the mean \pm standard deviation. Statistical comparisons between groups were made by analysis of variance with the Student-Newman-Keul post hoc test or Student's t-test. $\mathrm{P}<0.05$ was considered to indicate a statistically significant difference.

\section{Results}

YAP expression levels are positively associated with the drug resistance of LOVO cells to TAX. To investigate the association of YAP levels and the resistance of LOVO cells to TAX, the expression levels of YAP were determined in LOVO cells and LOVO/TAX cells. Compared with those in LOVO cells, the YAP expression levels were significantly higher in LOVO/TAX cells at the mRNA and the protein level (Fig. 1).

Next, in order to determine the effect of YAP expression on the sensitivity of LOVO/TAX cells to TAX, YAP was inhibited by YAP siRNA. The results indicated that the expression levels of YAP were significantly inhibited in LOVO/TAX 
A

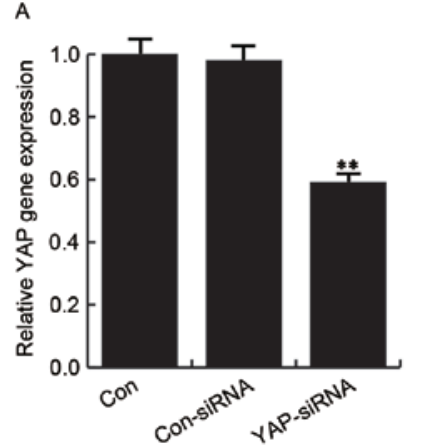

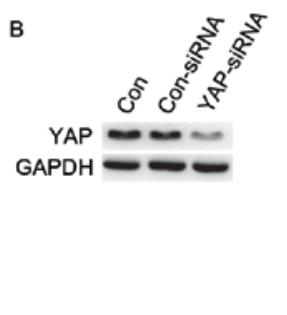

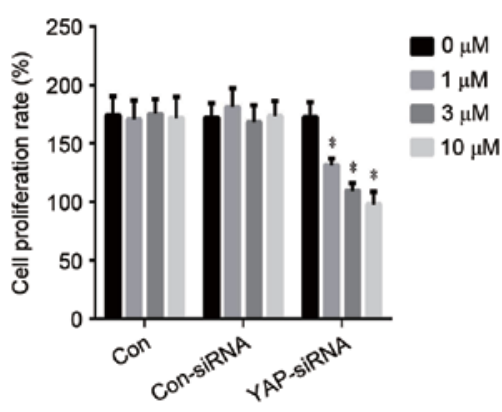

Figure 2. YAP inhibition enhances drug sensitivity of LOVO/TAX cells to taxol. (A) Relative YAP mRNA expression in LOVO/TAX cells. (B) Protein expression levels of YAP in LOVO/TAX cells. (C) Proliferation ability in different groups. Values are expressed as the mean \pm standard deviation. ${ }^{*} \mathrm{P}<0.05$; ${ }^{* *} \mathrm{P}<0.01$ vs. Con. YAP, Yes-associated protein; LOVO/TAX, taxol-resistant LOVO cell line; Con, control; siRNA, small interfering RNA.

A

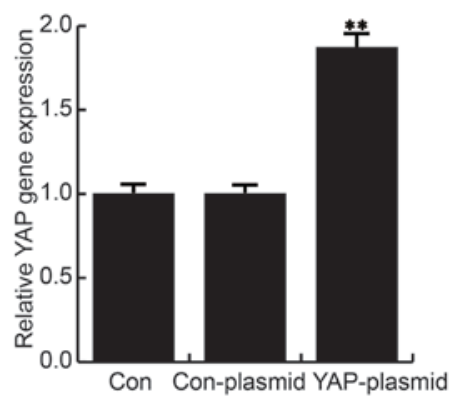

C

B

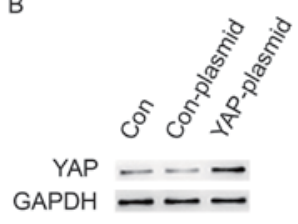

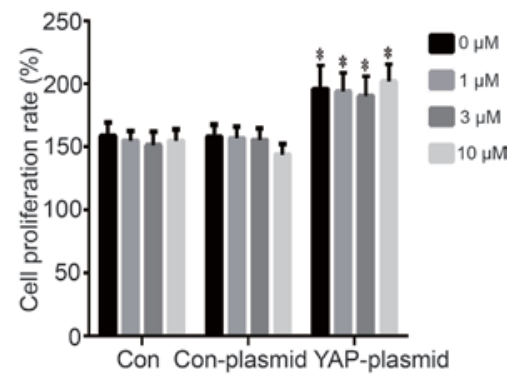

Figure 3. YAP overexpression inhibits drug sensitivity of LOVO cells to taxol. (A) Relative YAP mRNA expression in LOVO/TAX cells. (B) Protein expression levels of YAP in LOVO/TAX cells. (C) Proliferation ability in different groups. Values are expressed as the mean \pm standard deviation. ${ }^{*} \mathrm{P}<0.05$; ${ }^{* *} \mathrm{P}<0.01$ vs. Con. YAP, Yes-associated protein; LOVO/TAX, taxol-resistant LOVO cell line; Con, control.

cells by YAP siRNA at the mRNA and the protein level (Fig. 2A and B) and YAP inhibition notably enhanced the sensitivity of LOVO/TAX cells to TAX (Fig. 2C).

In addition, the effect of YAP overexpression on the sensitivity of LOVO cells to TAX was examined and the results indicated that YAP overexpression significantly reduced the sensitivity of LOVO cells to TAX (Fig. 3).

VP increases the sensitivity of LOVO/TAX cells to TAX by downregulating YAP expression. Since VP has been recognized as an effective YAP inhibitor, the present study assessed whether VP increased the sensitivity of LOVO/TAX cells to TAX by inhibiting YAP. The results indicated that TAX $(3 \mu \mathrm{M})$ had no significant influence on YAP expression, while VP inhibited YAP expression in a dose-dependent manner and YAP expression was inhibited by VP and TAX combined. Furthermore, at the highest concentration of VP, the expression of YAP was further decreased by TAX addition (Fig. 4).

The results also indicated that Bcl-2 family proteins were involved in the effect of VP on LOVO/TAX cells. Western blot analysis suggested that TAX $(3 \mu \mathrm{M})$ had no influence on the $\mathrm{Bcl}-2 / \mathrm{Bax}$ ratio, while VP reduced the $\mathrm{Bcl}-2 / \mathrm{Bax}$ ratio in a dose-dependent manner and the Bcl-2/Bax ratio was also inhibited by VP and TAX in combination. The addition of VP enhanced the sensitivity of LOVO/TAX cells to taxol (Fig. 4).

As YAP is overexpressed in the TAX-resistant cell subline LOVO/TAX, it was tested whether YAP inhibition by VP increased the sensitivity of LOVO/TAX cells to TAX. The

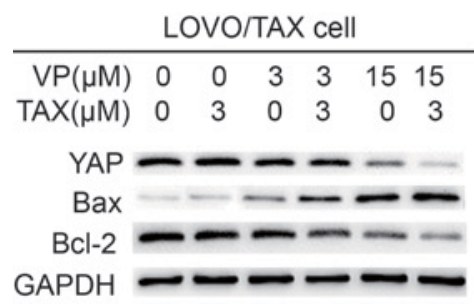

Figure 4. Effect of VP or/and TAX on YAP, Bax and Bcl-2 expression. Western blot analysis was performed to determine the influence of VP or/and TAX on YAP, Bax and Bcl-2 protein expression in LOVO/TAX cells. LOVO/TAX, taxol-resistant LOVO cell line; VP, verteporfin; TAX, taxol; YAP, Yes-associated protein; Bcl-2, B-cell lymphoma 2; Bax, Bcl-2-associated $\mathrm{X}$ protein

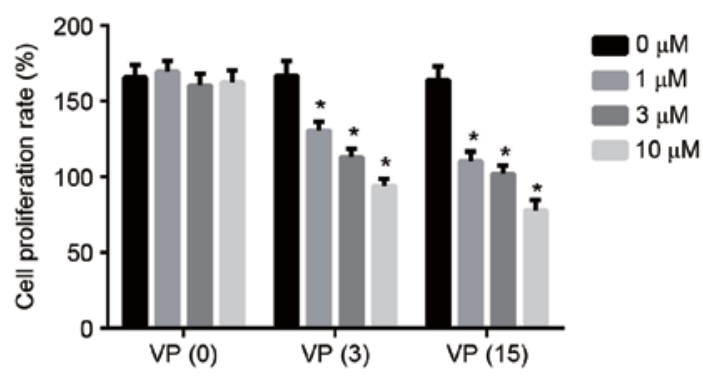

Figure 5 . VP $(0,3$ or $15 \mu \mathrm{M})$ enhances the sensitivity of LOVO/TAX cells to TAX $(0,1,3$ or $10 \mu \mathrm{M})$. An MTT assay was performed to detect LOVO/TAX cell proliferation ability after treatment with VP or/and TAX. Values are expressed as the mean \pm standard deviation. ${ }^{*} \mathrm{P}<0.05$ vs. Con. LOVO/TAX, TAX-resistant LOVO cell line; Con, control; VP, verteporfin; TAX, taxol. 
MTT assay revealed that the increased TAX concentration had no effect on cell proliferation in the $0 \mu \mathrm{M}$ VP group. However, decreased cell proliferation was observed in VP $(3$ and $5 \mu \mathrm{M})$ groups. Therefore, VP enhanced the sensitivity of LOVO/TAX cells to taxol. The results also revealed that VP alone had no impact on cell proliferation in the $0 \mu \mathrm{M}$ TAX group (Fig. 5).

\section{Discussion}

TAX is an anti-microtubule drug, which may be used in the treatment of a variety of tumors by promoting tubulin polymerization, inhibiting depolymerization, maintaining tubulin stability and inhibiting cell mitosis. As a novel anti-tumor drug, TAX and other microtubule-targeting drugs, have been frequently applied in chemotherapy in recent years. At present, TAX is used for treating ovarian, breast, lung and gastrointestinal cancer, as well as leukemia and has produced encouraging results (17). However, drug resistance has always been the bottleneck of its clinical application. For instance, in gastric cancer treatment, TAX, whether applied as a single-agent or combination therapy, may provide a benefit for patients (18). However, $30 \%$ of patients do not benefit from TAX treatment. Therefore, it is critical to explore novel strategies to reverse or mitigate drug resistance.

A previous study reported that overexpression of YAP promotes TAX resistance in ovarian cancer cells (19). Pan et al (10) reported that YAP was more strongly expressed in HCT-8/T than in HCT-8 cells and that TAX resistance was correlated with the level of YAP expression. Accordingly, YAP may have a similar role in the LOVO colon cancer cell line. The present study revealed that, compared with the LOVO cell line, the expression levels of YAP were significantly higher in LOVO/TAX cells. YAP inhibition notably enhanced the sensitivity of LOVO/TAX cells to TAX and ectopic overexpression of YAP significantly reduced the sensitivity of LOVO cells to TAX. These results indicated that overexpression of YAP in colon cancer LOVO cells induces resistance to TAX and that inhibition of YAP reverses it. This result was consistent with that of the previous study.

VP is widely and safely used in the treatment of neovascular macular degeneration as well as certain human tumors (15). VP represses YAP expression without light activation by preventing the YAP-TEAD growth pathway. Therefore, VP may reverse the drug resistance caused by YAP overexpression. The results of the present study suggested that VP inhibited YAP expression in a dose-dependent manner and dose-dependently increased the sensitivity of LOVO/TAX cells to TAX. These results revealed that VP reverses the drug resistance caused by YAP overexpression.

In conclusion, the present study indicated that VP treatment enhanced the sensitivity of LOVO/TAX cells to TAX and thus reversed drug resistance, through inhibiting YAP expression.

\section{Acknowledgements}

Not applicable.

\section{Funding}

No funding was received.

\section{Availability of data and materials}

The datasets used and/or analyzed during the current study are available from the corresponding author on reasonable request.

\section{Authors' contributions}

GS contributed to study design, data collection and analysis. $\mathrm{HaW}$ and $\mathrm{HH}$ were responsible for data collection and analysis. JG and HuW performed the literature search and analysis, and contributed to the acquisition of data. All authors collaborated to interpret the results and write the manuscript.

\section{Ethics approval and consent to participate}

Not applicable.

\section{Consent for publication}

Not applicable.

\section{Competing interests}

The authors declare that they have no competing interests.

\section{References}

1. Torre LA, Bray F, Siegel RL, Ferlay J, Lortet-Tieulent J and Jemal A: Global cancer statistics, 2012. CA Cancer J Clin 65: 87-108, 2015

2. McCulloch M, Broffman M, van der Laan M, Hubbard A, Kushi L, Abrams DI, Gao J and Colford JM: Colon cancer survival with herbal medicine and vitamins combined with standard therapy in a whole-systems approach: Ten-year follow-up data analyzed with marginal structural models and propensity score methods. Integr Cancer Ther 10: 240-259, 2011.

3. Liu AM, Xu Z and Luk JM: An update on targeting Hippo-YAP signaling in liver cancer. Expert Opin Ther Targets 16: 243-247, 2012.

4. Hong L, Cai Y, Jiang M, Zhou D and Chen L: The Hippo signaling pathway in liver regeneration and tumorigenesis. Acta Biochim Biophys Sin (Shanghai) 47: 46-52, 2015.

5. Zhang X, George J, Deb S, Degoutin JL, Takano EA, Fox SB; AOCS Study group; Bowtell DD and Harvey KF: The Hippo pathway transcriptional co-activator, YAP, is an ovarian cancer oncogene. Oncogene 30: 2810-2822, 2011.

6. Su LL, Ma WX, Yuan JF, Shao Y, Xiao W and Jiang SJ: Expression of Yes-associated protein in non-small cell lung cancer and its relationship with clinical pathological factors. Chin Med J (Engl) 125: 4003-4008, 2012.

7. Xu MZ, Yao TJ, Lee NP, Ng IO, Chan YT, Zender L, Lowe SW, Poon RT and Luk JM: Yes-associated protein is an independent prognostic marker in hepatocellular carcinoma. Cancer 115: 4576-4585, 2009.

8. Zhang J, Xu ZP, Yang YC, Zhu JS, Zhou Z and Chen WX: Expression of Yes-associated protein in gastric adenocarcinoma and inhibitory effects of its konckdown on gastric cancer cell proliferation and metastasis. Int J Immunopathol Pharmacol 25: 583-590, 2012.

9. Zhang L, Yang S, Chen X, Stauffer S, Yu F, Lele SM, Fu K, Datta K, Palermo N, Chen Y and Dong J: The hippo pathway effector YAP regulates motility, invasion, and castration-resistant growth of prostate cancer cells. Mol Cell Biol 35: 1350-1362, 2015.

10. Pan W, Wang Q, Zhang Y, Zhang N, Qin J, Li W, Wang J, Wu F, Cao L and Xu G: Verteporfin can reverse the paclitaxel resistance induced by YAP over-expression in HCT-8/T cells without photoactivation through inhibiting YAP expression. Cell Physiol Biochem 39: 481-490, 2016.

11. Ivery MT and Le T: Modeling the interaction of paclitaxel with beta-tubulin. Oncol Res 14: 1-19, 2003. 
12. Joo Y, Schumacher B, Landrieu I, Bartel M, Smet-Nocca C, Jang A, Choi HS, Jeon NL, Chang KA, Kim HS, et al: Involvement of 14-3-3 in tubulin instability and impaired axon development is mediated by Tau. FASEB J 29: 4133-4144, 2015

13. Jinawath N, Vasoontara C, Yap KL, Thiaville MM, Nakayama K, Wang TL and Shih IM: NAC-1, a potential stem cell pluripotency factor, contributes to paclitaxel resistance in ovarian cancer through inactivating Gadd45 pathway. Oncogene 28: 1941-1948, 2009.

14. Zhang H, Ramakrishnan SK, Triner D, Centofanti B Maitra D, Gyorffy B, Sebolt-Leopold JS, Dame MK, Varani J, Brenner DE, et al: Tumor-selective proteotoxicity of verteporfin inhibits colon cancer progression independently of YAP1. Sci Signal 8: ra98, 2015.

15. BrodowskaK,Al-Moujahed A,Marmalidou A,MeyerZu HorsteM, Cichy J, Miller JW, Gragoudas E and Vavvas DG: The clinically used photosensitizer Verteporfin (VP) inhibits YAP-TEAD and human retinoblastoma cell growth in vitro without light activation. Exp Eye Res 124: 67-73, 2014.
16. Livak KJ and Schmittgen TD: Analysis of relative gene expression data using real-time quantitative PCR and the 2(-Delta Delta C(T)) method. Methods 25: 402-408, 2001.

17. Pei BX, Sun YS and Dong SH: Curative effect of Abraxane in patients with non-small-cell lung cancer and breast cancer. Chin Pharm J 46: 1851-1854, 2011.

18. Sakamoto J, Matsui T and Kodera Y: Paclitaxel chemotherapy for the treatment of gastric cancer. Gastric Cancer 12: 69-78, 2009.

19. Xia Y, Chang T, Wang Y, Liu Y, Li W, Li M and Fan HY: YAP promotes ovarian cancer cell tumorigenesis and is indicative of a poor prognosis for ovarian cancer patients. PLoS One 9: e91770, 2014. 